\title{
Confirmation of a New Metal-poor Globular Cluster in the Galactic Bulge*
}

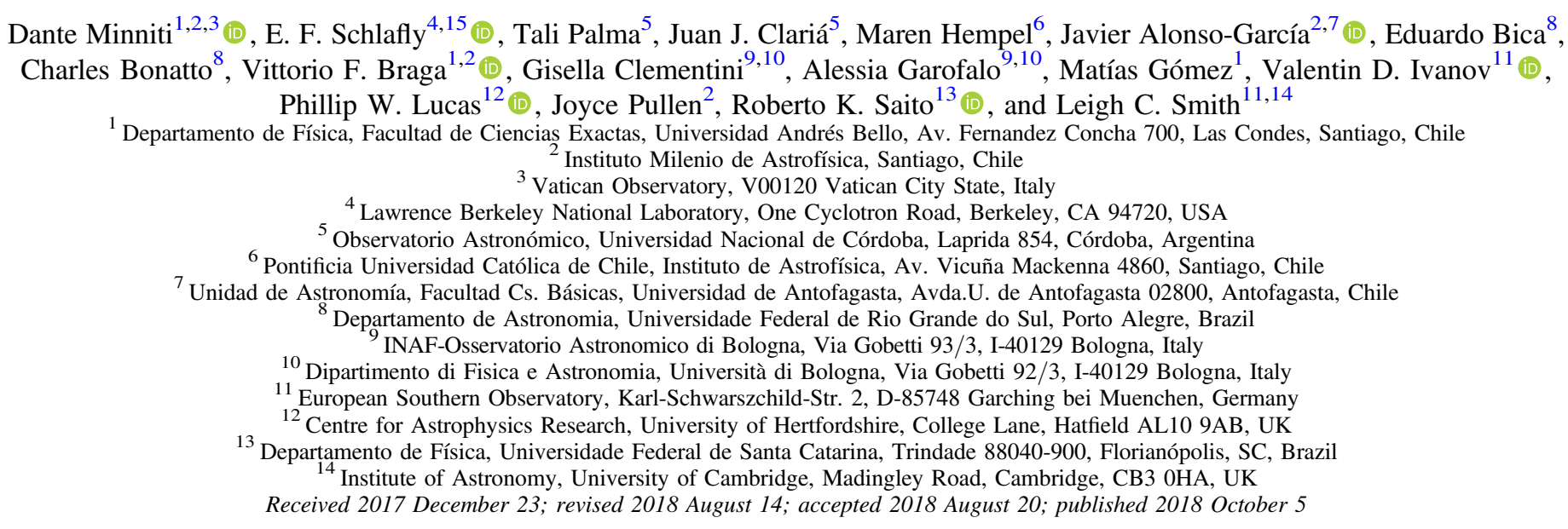

\begin{abstract}
We use deep near-IR photometry of the VISTA Variables in the Vía Láctea (VVV) Survey and deep DECam Plane Survey (DECaPS) optical photometry to confirm the physical reality of the candidate globular cluster (GC) Minni 22 , which is located in the Galactic bulge. This object, which was detected as a high density region in our maps of bulge red giants, is now confirmed as a real GC based on the optical and near-IR color-magnitude diagrams. We also recover three known fundamental mode (ab type) RR Lyrae stars within 2 arcmin of the cluster center. The presence of RR Lyrae stars also seems to confirm Minni 22 as a bonafide old and metal-poor GC. We estimate a cluster reddening $E(J-K s)=0.6 \mathrm{mag}$ and determine its heliocentric distance $D=7.4 \pm 0.3 \mathrm{kpc}$. The optical and near-IR color-magnitude diagrams reveal well-defined red giant branches in all cases, including a red giant branch bump at $K s=13.30 \pm 0.05$ mag. The comparison with theoretical isochrones yields a mean metallicity of $[\mathrm{Fe} / \mathrm{H}]=-1.3 \pm 0.3$ dex, and age of $t \sim 11.2 \mathrm{Gyr}$. This is a good example of a new low-luminosity $\left(M_{V}=-6.2 \mathrm{mag}\right) \mathrm{GC}$ found in the central bulge of the Milky Way. After discussing the different ways to confirm the existence of bulge GC candidates, we find that one of the best methods is to use the CMDs from the combination of the DECaPS + VVV photometries.
\end{abstract}

Key words: Galaxy: bulge - Galaxy: evolution - globular clusters: general globular clusters: individual (Minni 22)

Supporting material: machine-readable table

\section{Introduction: How to Confirm a Globular Cluster?}

Globular clusters (GCs) are very interesting astronomical objects because they represent the oldest populations of our Galaxy. Part of them have survived dynamical destruction processes and they have unique structural parameters, chemical compositions reflecting the first generation of stars, and their age distribution teaches us about the formation of the Milky Way. GCs can be used to develop and test models of stellar evolution and nucleosynthesis. However, they are relatively rare, containing only about $2 \%$ of the stars in the halo of our Galaxy (Suntzeff et al. 1991). The identification of new GCs (even low-luminosity ones) would allow us to significantly improve our knowledge of the Galactic GC population. The importance of the discovery of another GC should not be minimized. In fact, the Galactic GCs are much less numerous than the open clusters; for example, fewer than 200 of them are known to date in the entire Galaxy. Therefore, any new

\footnotetext{
* Based on observations collected at the European Organisation for Astronomical Research in the Southern Hemisphere under ESO programmes 179.B-2002 and 298.D-5048.

${ }^{15}$ Hubble Fellow.
}

discovery is important because each GC is a treasure of information and allows a variety of studies in the fields of dynamics, chemical composition, stellar populations, stellar evolution, stellar variability, and so on.

The metal-poor GCs in the Galactic bulge, in particular, are very interesting because they may represent the oldest stellar systems in our Galaxy (e.g., Barbuy et al. 2006, 2009). However, searching for GCs in the Galactic bulge is a hard task and new Galactic GCs in this region are very difficult to find due to both high stellar density and interstellar extinction. New cluster candidates that are identified in images by using visual inspection or automatic detection algorithms should exhibit a significant overdensity above the background. They should also be seen in both the optical and near-IR images, although this criterion should be less strict in the presence of high (background and foreground) stellar contamination. They should be more or less round, although this criterion can be relaxed in the presence of differential reddening. In addition, at the distance of the Galactic bulge, the GC sizes (half light radii) should be between 1 and 10 arcmin (White \& Shawl 1987; Chen \& Chen 2010). 
Confirming the presence of a GC in the Galactic bulge is also difficult. There is probably no unique recipe to decide that a candidate is a real GC. For example, confirming a GC located in the Galactic halo is very different from confirming a cluster located in the inner bulge. The requirements for the GCs located in the bulge should be much more stringent given the large and differential extinction, and the high foreground and background stellar contamination. To confirm the physical reality of a $\mathrm{GC}$, one or a few of the following methods can be used:

(1) Building a CMD and determining the cluster luminosity function (LF) to detect the features and sequences typical of GCs, such as the red giant branch (RGB), the turn-off (TO), the horizontal branch (HB), the red clump (RC) and the bump in the red giant branch (RGBB). This is a very powerful method but, in case of severe foreground and background contamination (like in the bulge or in low latitude disk fields), a decontamination method is recommended to statistically subtract the background from the CMD and from the LF (e.g., Bonatto et al. 2007; Palma et al. 2016). However, this procedure requires deep (passed the main-sequence TO) and high quality data (with small photometric errors and high spatial resolution).

(2) Using photometry from independent datasets, preferably in different passbands (e.g., a new IR cluster candidate can be confirmed with optical photometry, and vice versa). However, in case of very high interstellar extinction, confirmation by optical photometry would be practically impossible.

(3) Adjusting theoretical isochrones to the observed CMDs or comparing these CMDs with those of known GCs of similar metallicities.

(4) Detecting and using RR Lyrae variable stars that are representative of old and metal-poor stellar populations. These stars are frequently found not only in metal-poor GCs but also in the Galactic halo and bulge. They are excellent reddening and distance indicators. The presence of RR Lyrae variable stars in a stellar cluster guarantees that this is a GC. In the bulge fields, the concentration of RR Lyrae stars has to be larger than the background (the bulge RRL density varies from 80 to $440 \mathrm{RRL} / \mathrm{deg}^{2}$ from Pietrukowicz et al. 2015) and they have to be located at similar distances to be considered members of the same GC. However, this method cannot be used for metal-rich GCs, which generally do not contain RR Lyrae variables.

(5) Using proper motions (PMs) astrometrically or radial velocities (RVs) spectroscopically. The measurements of PMs and/or RVs can be used to decontaminate the CMDs to confirm the features and sequences typical of GCs. It is clear that this technique is only capable of helping if one can tell bulge PMs (or RVs) from GC PMs (or RVs), which depend on the cluster mean motion, and therefore in some cases it may require more precision than is so far available.

(6) Determining spectroscopically individual stellar abundances that yield the chemical footprint typical of GCs. However, this last method is costly in telescope time because it requires high dispersion spectroscopy and relatively high SNR.
It is worth pointing out that many of these techniques are vulnerable to the effects of variable extinction and reddening in similar ways, and accordingly are not really independent tests. For example, dust-related concerns will affect techniques 1, 2, 3 , and-to a lesser extent-technique 4 .

In this work we explore several of these procedures to confirm the physical reality of one of the bulge GC candidates that have recently been discovered by Minniti et al. (2017). We selected cluster Minniti 22 (Minni 22, for short) as one of the best candidates because of the presence of RR Lyrae variable stars in its field, as discussed later on. Minni 22 turns out to be a new GC that is not listed in any of the previously known GC catalogs. In particular, to confirm Minni 22 as a real GC, we combine data from two large surveys of the Galactic plane: the DECam Plane Survey (DECaPS) optical data (Schlafly et al. 2017) with the VISTA Variables in the Via Lactea (VVV) Survey near-IR data (Minniti et al. 2010).

\section{The GC Minni 22}

We use data from the VVV Survey (Minniti et al. 2010; Saito et al. 2012) that were acquired with the VIRCAM camera at the VISTA $4 \mathrm{~m}$ telescope at the ESO Paranal Observatory (Emerson \& Sutherland 2010) between years 2010 and 2016, and reduced at the Cambridge Astronomical Survey Unit (CASU) with the VIRCAM pipeline v1.3 (Irwin et al. 2004). The PSF photometry that we adopted to build the deep near-IR CMDs was made with DoPhot, following Alonso-García et al. (2015).

We also exploit the data obtained by the DECaPS Survey, which is a griz $Y$-band optical survey of the southern Galactic plane (Schlafly et al. 2017). This survey was carried out using the Dark Energy Camera at the $4 \mathrm{~m}$ telescope of the Cerro Tololo Inter-American Observatory. The DECaPS optical photometry is very deep, reaching past the main-sequence TO at the distance of the bulge for moderate reddenings $E(B-V) \sim 1.5 \mathrm{mag}$. Typical photometric depths are 23.7, $22.8,22.3,21.9$ and 21.0 mag in the griz $Y$-bands, respectively, with a seeing of around 1 arcsec. The DECaPS Survey covers the highly reddened regions of the Southern Galactic plane with $5>l>-120^{\circ}$ and $|b|<4^{\circ}$, including the cluster studied here.

Minniti et al. (2017) discovered 22 new GC candidates in the Galactic bulge using density maps that were made after performing appropriate cuts in the Wesenheit near-IR CMD. To seek confirmation of these clusters, we started by choosing the best candidate. First, we selected the clusters that have RR Lyrae stars within 1 arcmin from their respective centers. These clusters are: Minni 9, 11, 12, 20, and 22. They all have one RR Lyrae star within 1 arcmin. However, only one cluster, Minni 22, has two RR Lyrae stars within 1 arcmin. Further inspection revealed that this cluster has three RR Lyrae type ab (RRab) stars within 2 arcmin of its center. As will be discussed later, the magnitudes - and therefore the distances - of the RR Lyrae stars are consistent with cluster membership, which made Minni 22 the prime object for an investigation of its real GC nature.

Minni 22 is located at Equatorial coordinates R.A. = 17:48:51.4, decl. $=-33: 03: 40$ (J2000), and Galactic coordinates $l=356^{\circ} .8283, b=-2^{\circ} .7285$, in VVV tile $\mathrm{b} 289$, at an angular distance of about 4.5 from the Galactic center. In Figure 1, we show a density map of red giants (selected following Minniti et al. 2017) covering a field of view of 

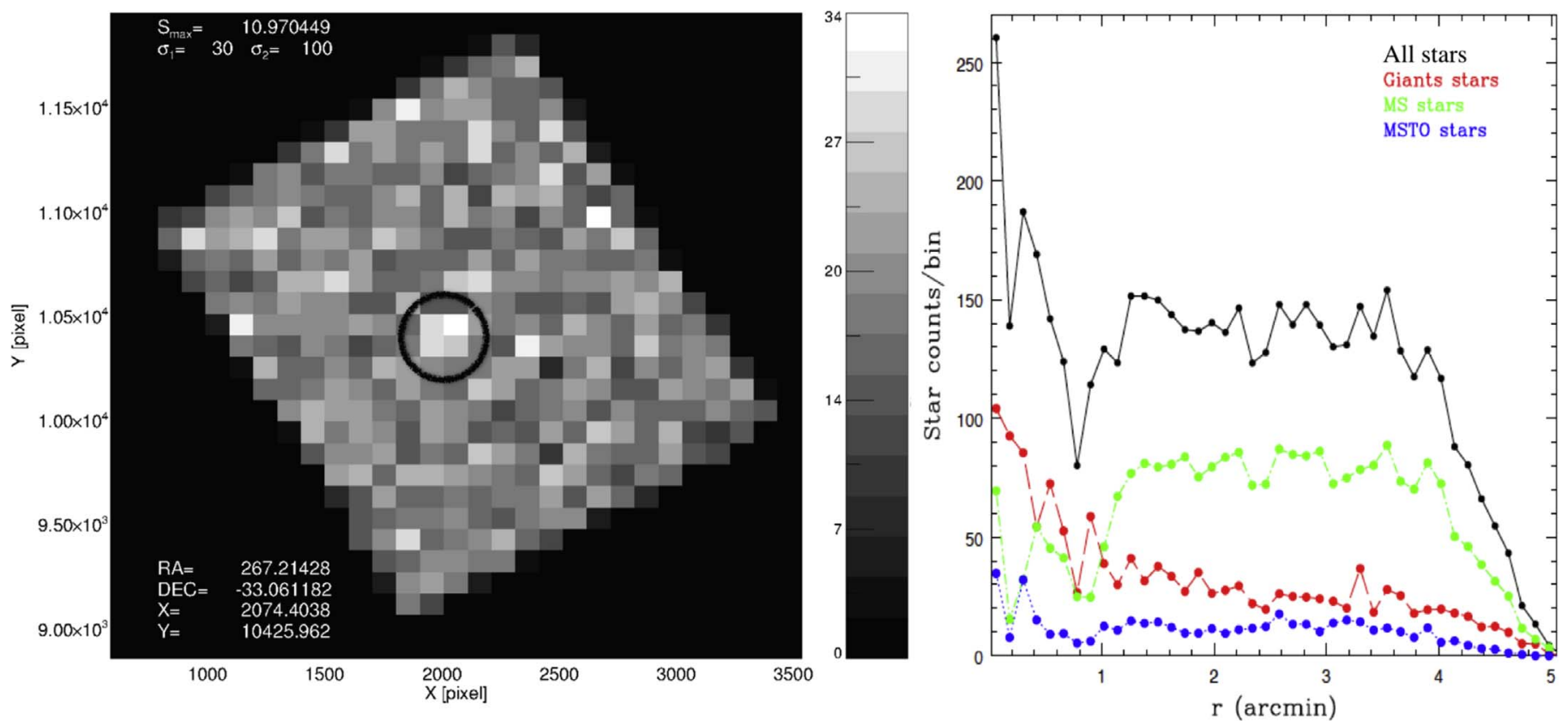

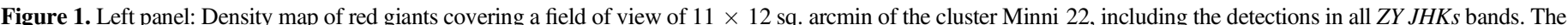

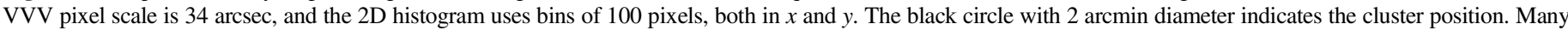

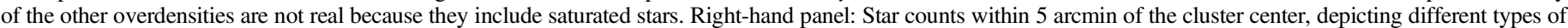

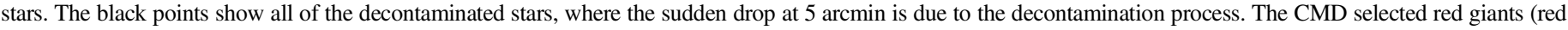
points) show better the cluster concentration, while the fainter stars (blue points) and foreground disk main-sequence (MS) stars (green) show no concentration.

$11 \times 12$ sq. arcmin of this cluster. This map clearly shows an overdensity in the cluster region. This detection has a significance of 11.0 according to the test of Koposov et al. (2007). A similar overdensity of red giants is seen in the maps of the optical sources in the DECaPS images. However, the other overdensities are not real because they correspond to saturated stars. After a test to confirm false positives of similar significance, we eliminated the more saturated stars and we confirmed that the only real overdensity was at the cluster's location.

Minni 22 is one of the smallest clusters found by Minniti et al. (2017), for which we measure a half-light-radius of $r_{h} \sim 1.1 \pm 0.3$ arcmin, although we note that this is uncertain because of the high number of foreground and background sources. A finding chart of $3.8 \times 3.8$ sq. arcmin centered on Minni 22 is shown in Figure 2. Due to the high stellar density, the cluster can be barely seen in the optical and near-IR images. Therefore, its size is poorly defined but it is certainly at the low end of the size distribution for Galactic GCs (White \& Shawl 1987; Chen \& Chen 2010).

\section{RR Lyrae and Other Variable Stars in Minni 22}

There are 3 RRab stars in the field of Minni 22, located within 2 arcmin of its center. These RRab variables, which are listed in Table 1, were recently detected by the Optical Gravitational Lensing Experiment (OGLE) Survey (Pietrukowicz et al. 2015). Table 1 presents star identifications, photometry in the various filters (VI from OGLE and ZYJHK from VVV) and distance moduli. The OGLE light curves needed to be phase corrected to interpolate the sample to the $J$-band. However, after this correction, neither the mean magnitudes nor the results changed. All three RRab stars have a relatively narrow spread in periods, magnitudes and colors, indicating that they have similar reddenings and that they are all located at approximately the same distance. The average RR Lyrae surface density in the region is found to be $0.07 \mathrm{RRab} /$ sqarcmin in the surrounding field, while the RR Lyrae density centered in the cluster is 3.5 times higher; that is, $0.24 \mathrm{RRab} /$ sqarcmin. Despite the small statistics sample, there is a significant RR Lyrae excess. To be more quantitative, the periods are slightly different, with $P_{3181}=$ 0.51 days, typical of an $0 \mathrm{oI}$ population, $P_{3178}=0.59$ days, typical of an 0o-intermediate population, and $P_{3258}=0.62$ days, typical of an 0oII population. Therefore, the Oosterhoff class of this cluster is uncertain (Oosterhoff 1939, see also Catelan $\&$ Smith 2015). We note that the total color ranges are $\Delta(I-K s)=0.16, \Delta(J-K s)=0.26$, and $\Delta(V-I)=0.16$. These ranges are narrow enough, except for $\Delta(J-K s)$, which can be explained considering that there are numerous multiple observations in the $K s$-band, while the $J$-band is the result of a single epoch observation.

We list the distance moduli of the three RR Lyrae in the last column of Table 1, which were computed using the PLZ relation from Muraveva et al. (2015). Based on these distance moduli, we estimate their mean value to be $(m-M)_{0}=$ $14.3 \pm 0.08$, equivalent to $D=7.3 \pm 0.3 \mathrm{kpc}$. A different PLZ relation from Navarrete et al. (2017) yields a slightly larger mean distance modulus $(m-M)_{0}=14.40 \pm 0.08$ or $D=7.6 \pm 0.3 \mathrm{kpc}$. Therefore, we adopt a mean RRab distance of $D=7.4 \pm 0.3 \mathrm{kpc}$. However, to quantify the possible differences, if we consider only OGLE-BLG-RRLYR-3181 and 3178 (the closest), then $m-M=14.25 \pm 0.08$ from Muraveva et al. (2015) and $14.35 \pm 0.08$ from Navarrete et al. (2017) are obtained. If we consider only OGLE-BLG-RRLYR3181 and 3258 (with periods of 0.58 and 0.62 days), then $m-M=14.35 \pm 0.07$ from Muraveva et al. (2015) and $14.44 \pm 0.07$ from Navarrete et al. (2017) are yielded.

Figure 3 shows the location of the three newly discovered RR Lyrae stars superimposed on the CMD of the bulge RRab stars from Pietrukowicz et al. (2015). This figure implies that 


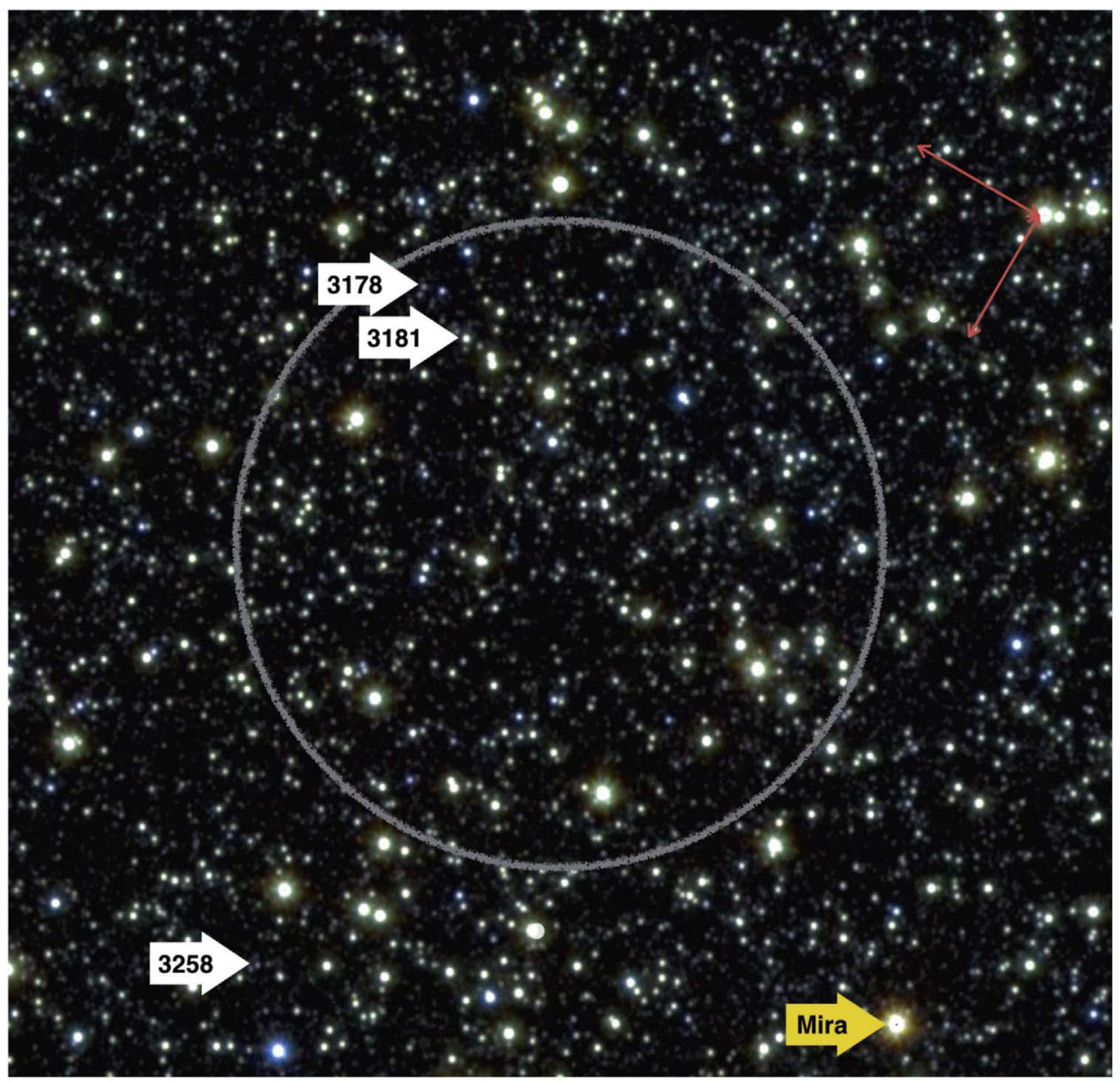

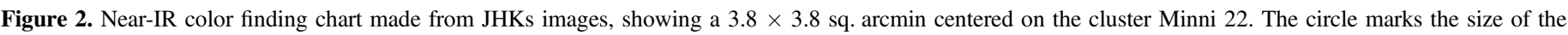

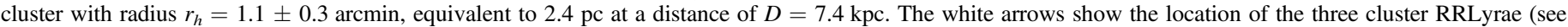

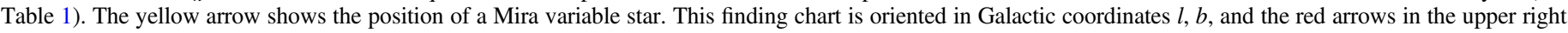
show the orientation of Equatorial coordinates R.A., decl.

Table 1

RR Lyrae Type ab in the Globular Cluster Minni 22

\begin{tabular}{|c|c|c|c|c|c|c|c|c|c|c|}
\hline ID & R.A. 2000 & Decl.2000 & long. & lat. & Period (day) & $V$ & $I$ & $J$ & $K s$ & $(m-M)_{0}$ \\
\hline OGLE-BLG-RRLYR-3181 & $17: 48: 49.38$ & $-33: 03: 03.0$ & 356.8336 & -02.7171 & 0.51239463 & 19.57 & 17.08 & 15.08 & 14.33 & 14.23 \\
\hline OGLE-BLG-RRLYR-3178 & $17: 48: 49.02$ & $-33: 02: 52.1$ & 356.8355 & -02.7144 & 0.58989198 & 19.40 & 16.92 & 15.23 & 14.36 & 14.35 \\
\hline OGLE-BLG-RRLYR-3258 & 17:49:00.30 & $-33: 03: 35.7$ & 356.8454 & -02.7545 & 0.62030367 & 19.27 & 16.94 & 15.36 & 14.35 & 14.32 \\
\hline
\end{tabular}

the three RRab stars that are listed in Table 1 are slightly brighter on average (and are therefore located closer) than the bulk of the RRab variable stars in the bulge. Considering the known RR Lyrae stars in the bulge, we can estimate the expectation for three $R R$ ab stars to fall within a 2 arcmin radius and to have similar distance moduli $\left(\Delta \Delta(m-M)_{0} \mid<0.1 \mathrm{mag}\right)$. Poisson statistics and Montecarlo simulations yield a very low probability of this occurrence $(P<1 \%)$. Consequently, the fact that the tight group of three RR Lyrae stars lie at consistent distances is the most convincing proof that we are dealing with a real GC.

There is another RR Lyrae type c (RRc) candidate in the field of Minni 22, with period $P=0.25906458$ days, located at about 2 arcmin away from the cluster center. This is OGLEBLG-RRLYR-3266, situated at R.A. = 17:49:01.31, decl. = $-33: 02: 11.7$ (J2000). However, this object is much too faint to be a cluster member. With measured magnitudes $V=19.64$,
$I=17.49, J=15.88$, and $K s=15.14$, it should be much more distant than Minni 22. We then classify this RRc star as a background variable star.

There are also a couple of Mira variable stars located $\sim 3$ arcmin due South, and $\sim 3$ arcmin due East from the cluster center, respectively. These Miras are 2MASS J174902193302189 and OGLE-BLGLPV-47292, and they may also be associated with the cluster. Unfortunately, these stars are too bright $(K s<8)$, and appear saturated in the VVV and in the DECaPS images. Although these Mira stars in the CMD are not located far from the cluster RGB, this fact in itself is not enough to confirm cluster membership. Consequently, RVs are needed. In addition, six variable stars are also found within 2 arcmin of the GC center, which are classified as semiregular variables. These stars are: OGLE-BLGLPV-47165, OGLEBLGLPV-46992, OGLE-BLGLPV-47119, OGLE-BLGLPV48550, and OGLE-BLGLPV-46147. Like the Mira stars, these 


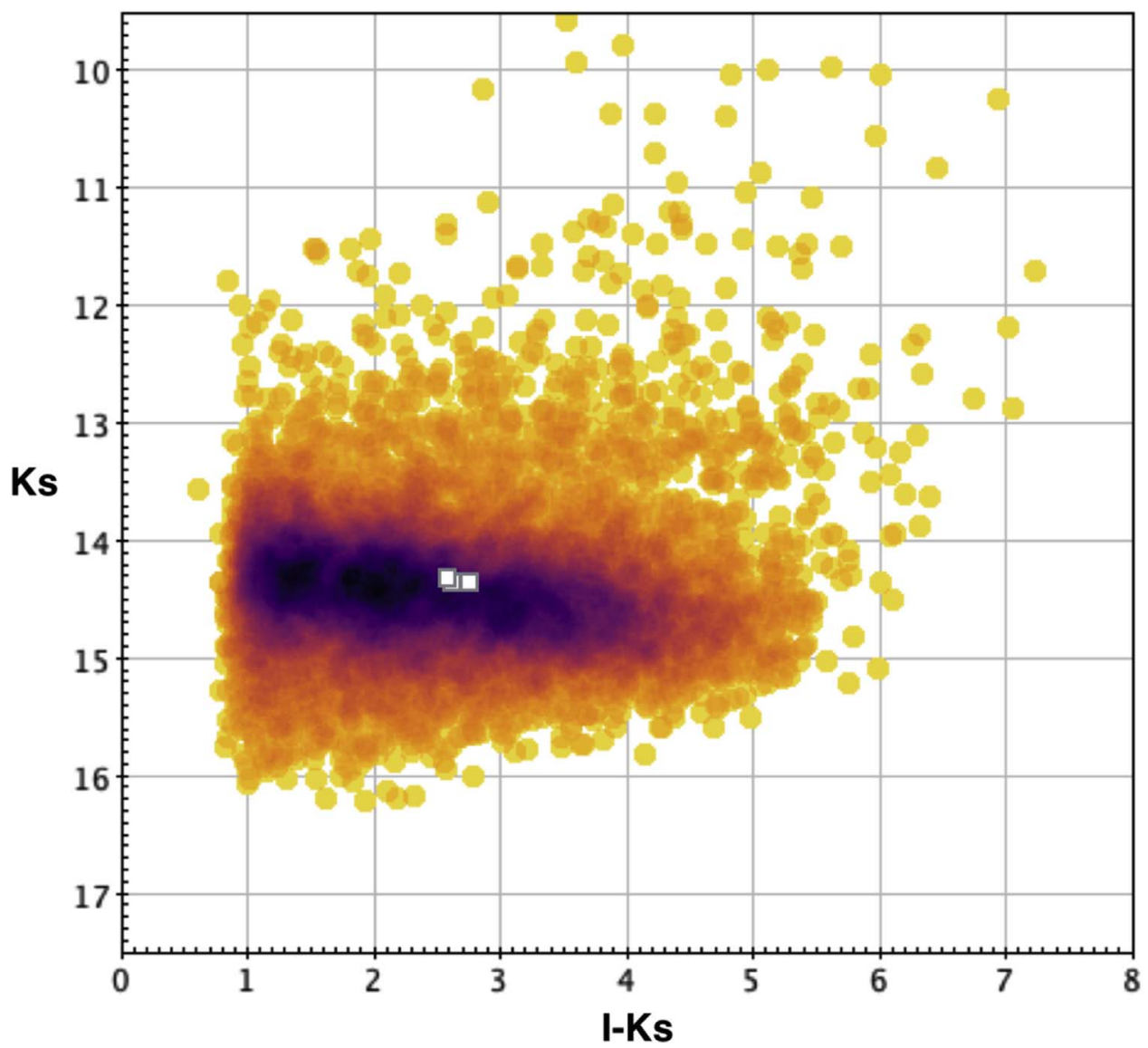

Figure 3. CMD for Galactic bulge RR Lyrae type ab from Pietrukowicz et al. (2015), showing the location of the three RRab cluster members. This figure shows that their distance is consistent with the peak of the bulge RRab distribution. The density scale is arbitrary.

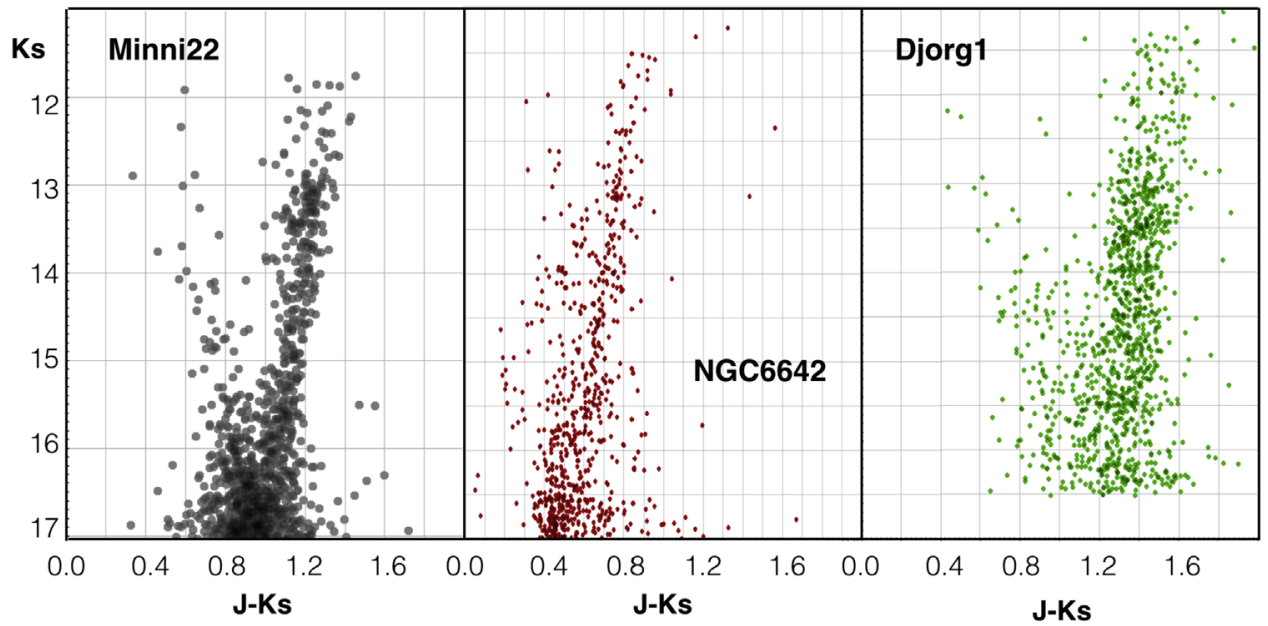

Figure 4. Near-IR CMD for the globular cluster Minni 22 (left-hand) along with the comparison clusters NGC 6642 (middle) and Djorg1 (right-hand). These have been obtained from PSF photometry of the VVV Survey images following Alonso-García et al. (2015). Note the resemblance of Minni 22 with NGC 6642, which shows an extended HB and a clear RGBB.

variables also appear saturated in the VVV and DECaPS images. Consequently, based on our photometric data, they could not be confirmed as cluster members.

\section{Near-IR CMDs and Comparison with Known GCs}

Figure 4 shows the near-IR CMD for the GC Minni 22 compared with the CMDs of the known Galactic GCs NGC 6642 and Djorg 1. Minni 22 is close in projection (17.5 arcmin away) to the GC Djorg 1 that is located at Galactic coordinates $l=356^{\circ} .67, b=-2^{\circ} .48$ in the VVV tile b303. Minni 22 is located in the bulge at $D=7.4 \mathrm{kpc}$, while Djorg 1 is at $D=12.0 \mathrm{kpc}$ (Harris 2010). Hence, both clusters are physically unrelated.

According to the CMDs, cluster Minni 22 appears to be significantly reddened with $E(J-K s)=0.6$ mag. From the existing maps, we obtain a near-IR extinction $A_{K s}=0.47 \mathrm{mag}$, adopting the field extinction of Schlafly \& Finkbeiner (2011), 


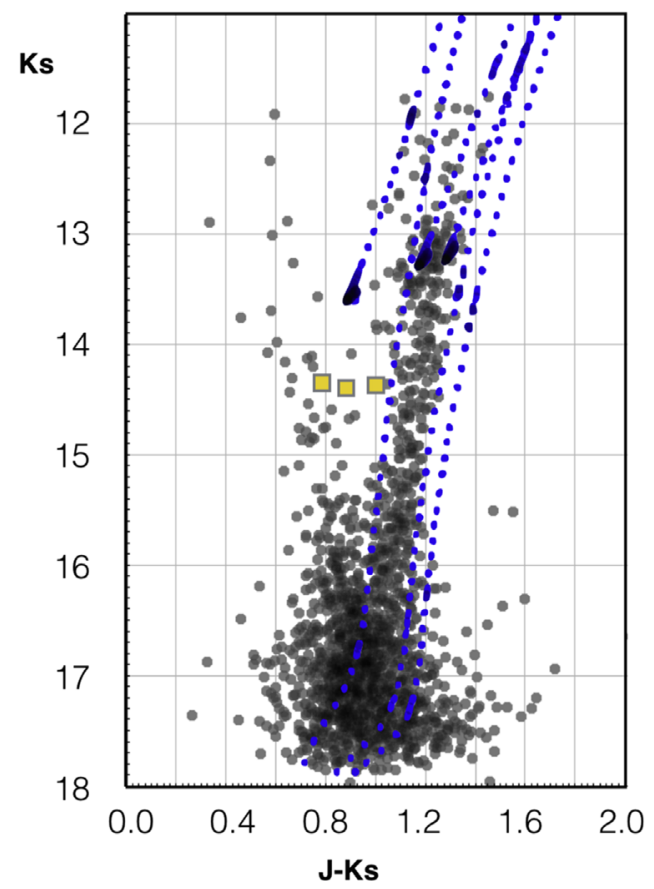

Figure 5. Near-IR CMD for the globular cluster Minni 22 compared with three selected PARSEC theoretical isochrones (Bressan et al. 2012; Marigo et al. 2017). The isochrones that are shown correspond to an age of $t=11.2$ Gyr, and metallicities $z=0.0001,0.0008$, and 0.02, from left to right. These isochrones have been shifted to fit the cluster RGB. The three RRab found to be cluster members are plotted with big yellow squares.

who use the extinction curve from Fitzpatrick (1999). For comparison, from the color excess maps by Gonzalez et al. (2012), and assuming the extinction law by Alonso-García et al. (2017), in this field the extinction is $A_{K s}=0.26 \mathrm{mag}$. The choice of extinction is one of the major sources of error in the distance determination that is made here. These differences arise from the various methods used and also from the slope of the adopted extinction law (e.g., the extinction slope of AlonsoGarcía et al. 2017 is steeper than those of Cardelli et al. 1989 and the Fitzpatrick 1999; therefore, producing less infrared extinction per optical extinction). Nonetheless, the interstellar extinction for Minni 22 is clearly smaller than that of GC Djorg $1\left(A_{K}=1.58 \mathrm{mag}\right)$. This is the reason why the near-IR CMD of Minni 22 looks cleaner than that of Djorg 1, which is also a metal-poor GC with $[\mathrm{Fe} / \mathrm{H}]=-1.58 \mathrm{dex}$ (Harris 2010; Valenti et al. 2010).

Minni 22 appears to be metal-poor based on the morphology of the CMD, which is very similar to that of the known GC NGC 6642 with $[\mathrm{Fe} / \mathrm{H}]=-1.3 \pm 0.2 \mathrm{dex}$ (Minniti 1995a; Barbuy et al. 2006; Kim et al. 2006; Harris 2010; Valenti et al. 2010), as shown in Figure 4. We note that even if the metallicity is not the only factor shaping the CMD of a GC, it is the major one and we also note that for our purposes it is fine to neglect other factors such as Helium abundance, rotation, etc. Using the CMDs, we can measure the heliocentric distances differentially with respect to the selected comparison clusters that are also observed by the VVV Survey. We adopted a distance $D_{\mathrm{NGC} 6642}=8.1 \mathrm{kpc}$ for the comparison cluster NGC 6642 (Harris 2010). Figure 4 shows that the RGBB of Minni 22 has a similar magnitude to the RGBB of NGC 6642. The RGBB in the near-IR CMDs of Minni 22 is well defined. Its mean magnitudes and colors are: $K s_{\mathrm{RGBB}}=13.30 \pm$ $0.05, \quad J-K s=1.22 \pm 0.02$, and $Y-K s=2.07 \pm 0.03$.
The RGBB of NGC 6642 has $K s_{\text {RGBB }}=13.20 \pm 0.10$ mag (Kim et al. 2006). Even though Minni 22 (with $A_{K s}=$ $0.47 \mathrm{mag}$ ) is more reddened than NGC 6642 (with $A_{K s}=$ $0.12 \mathrm{mag}$ ), we can conclude from the CMDs of Figure 4 that these two clusters should both be located in the Galactic bulge, which is consistent with the distance measured using the RR Lyrae, $D=7.4 \pm 0.3 \mathrm{kpc}$.

\section{Comparison with Theoretical Isochrones}

It is important to test if there is consistency between the theoretical isochrones for different combinations of optical and near-IR CMDs. This is the case for cluster Minni 22, where we have found that the isochrone for an old and metal-poor population fits well in all of the different CMDs.

Figure 5 shows the near-IR CMD for the GC Minni 22 compared with three selected PARSEC theoretical isochrones (Bressan et al. 2012; Marigo et al. 2017). ${ }^{16}$ The isochrones correspond to an age of $t=11.2 \mathrm{Gyr}$, and the metallicities are $z=0.0001,0.0008$, and 0.02 , from left to right. These isochrones were shifted to fit the cluster RGB. The three RRab stars members of this GC are also indicated (yellow squares), and they are fainter and bluer than the RGBB in this CMD, which is expected if they lie in the extended $\mathrm{HB}$ of the cluster.

We cannot accurately measure the cluster metallicity using the slope of the RGB (as prescribed by Valenti et al. 2004) because there are few stars and the upper part of the RGB is saturated in our photometry. However, the fit to the theoretical isochrones gives a good indication of its metallicity.

We decided to apply a statistical decontamination procedure to obtain a cleaner CMD. The diagrams of the $\mathrm{GC}$ region, as well as the comparison fields can be seen in Figure 6, in the upper left-hand and right-hand panels, respectively. Based on these CMDs, we calculate the contamination statistics for each $\mathrm{CM}$ space bin and we select a portion of sources at random to be cluster members as a function of this. The bottom right-hand panel of Figure 6 shows the GC region LF compared with with the LF of the field region. Even though they are similar, there is a clear excess of red cluster giants. The resulting decontaminated near-IR CMD of Minni 22 can be seen in the bottom lefthand panel and it reaches the main-sequence TO at $K s \sim$ $16.7 \mathrm{mag}$. Clearly, the background region has more differential extinction than the cluster region. This effect varies depending on which control region we choose. However, as revealed by the extensive comparisons made using numerous a dozen fields at different locations, the same effect is found in all of the surrounding background regions. Despite the higher spread in reddening, when the CMD of the cluster is superimposed over that of the background, the main CMD features seem to be well correlated with nearly similar extinctions.

We then fitted the Bressan theoretical set of isochrones, which offer the benefit of varying the metallicity in almost a continuous way. The isochrone that best fits the decontaminated CMD is for $Z=0.00095$ and $\log t=10.05$, and therefore we adopt the cluster metallicity of $[\mathrm{Fe} / \mathrm{H}]=-1.3 \pm$ $0.03 \mathrm{dex}$, and an age $t=11.2 \mathrm{Gyr}$. Caution with metallicity degeneracy should be taken, pending spectroscopic metallicity determinations. We also analyzed the LF and found that the clump is well defined at $K s=13.30 \pm 0.05$ mag. Based on the

\footnotetext{
16 Throughout the text, we use the PARSEC v1.28 isochrones for DECam ugrizY (ABmags) + VISTA ZY JHKs (Vegamags) from http://stev.oapd.inaf. it/cgi-bin/cmd.
} 


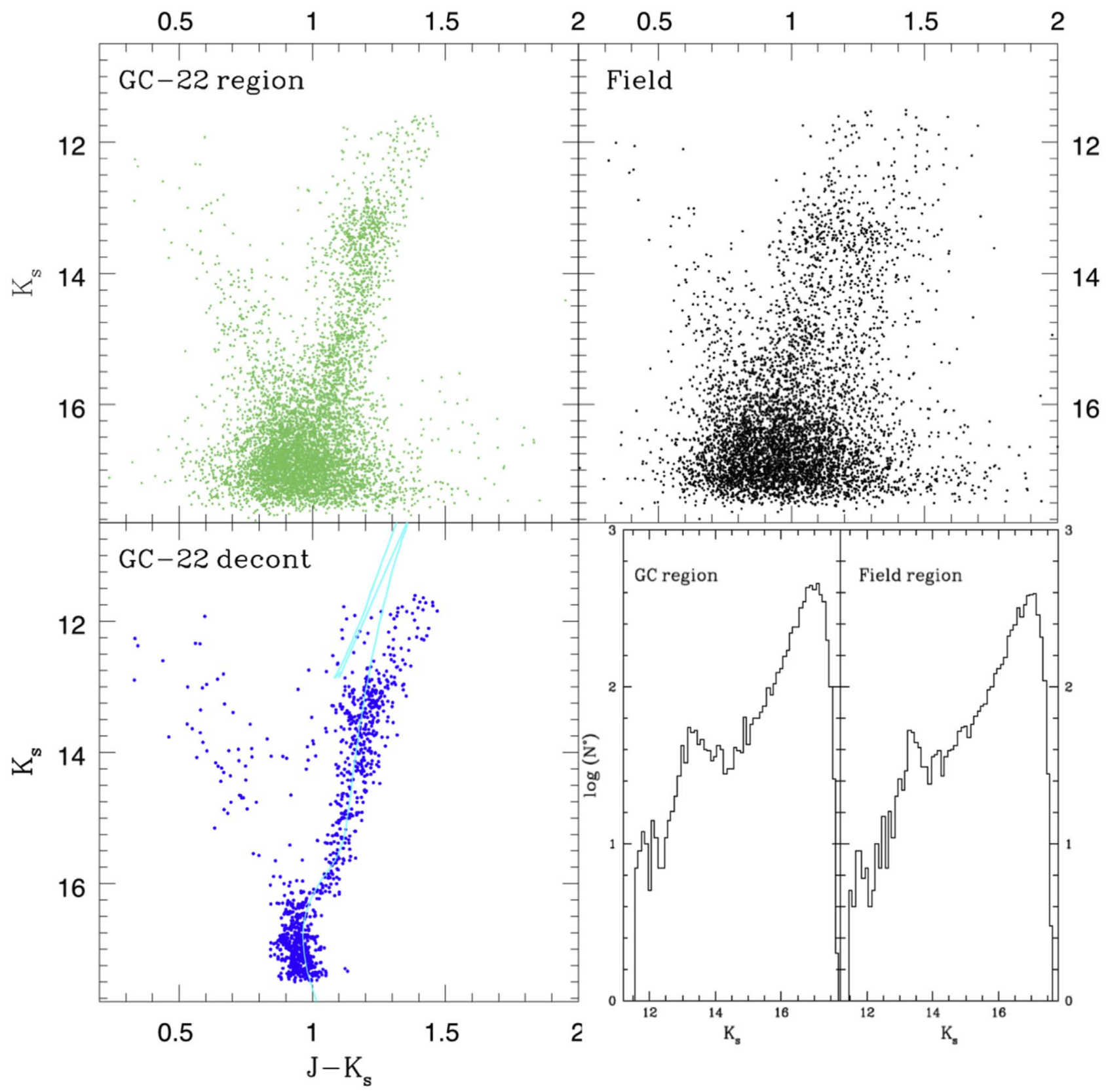

Figure 6. Near-IR CMD for a 2 arcmin sq. field centered in the globular cluster Minni 22 (top left-hand) compared with a background field of similar area located 5 arcmin away (top right-hand). The statistically decontaminated CMD (bottom left-hand) clearly shows the RGBB of the cluster. The shifts made for the isochrone fittings were: $E(J-K s)=0.69, A(K s)=0.35$ and $D M=13.9 \mathrm{mag}$. The insert shows the $K s$-band luminosity function for the cluster region vs the field region, respectively (bottom right-hand).

Table 2

The Combined VVV-DECaPS Multicolor Photometry

\begin{tabular}{|c|c|c|c|c|c|c|c|c|c|c|c|}
\hline ra_1_5 & dec_1_5 & mag_Z & mag_Y & mag_J & mag_H & mag_Ks & mag_g & mag_r & mag_i & mag_Y & mag_z \\
\hline 267.2260875 & -33.1386025 & 17.754 & 17.32 & 16.868 & 16.202 & 15.985 & 21.184 & 19.430 & 19.205 & 18.014 & 18.588 \\
\hline 267.2298145 & -33.1404604 & 19.247 & 18.794 & 18.194 & 17.583 & 17.315 & 23.0634 & 21.234 & 20.442 & 20.046 & 19.909 \\
\hline 267.2320693 & -33.1415155 & 18.165 & 17.676 & 17.005 & 16.403 & 16.145 & 22.055 & 20.092 & 19.232 & 18.451 & 18.667 \\
\hline 267.2171305 & -33.1340714 & 18.499 & 17.952 & 17.346 & 16.664 & 16.46 & 22.353 & 20.460 & 19.596 & 18.754 & 19.009 \\
\hline 267.2268224 & -33.1389 & 19.682 & 19.361 & 18.638 & 18.024 & 17.676 & 23.390 & 21.772 & 20.764 & 20.305 & 20.407 \\
\hline
\end{tabular}

(This table is available in its entirety in machine-readable form.)

comparison with the isochrone fittings, we conclude that Minni 22 is an old and metal-poor GC, which is consistent with the presence of the RR Lyrae variable stars.
The decontaminated LF after accounting for the blue stars (with $J-K s<0.9 \mathrm{mag}$, belonging to the foreground disk) also allows us to estimate the GC total luminosity. Simply 

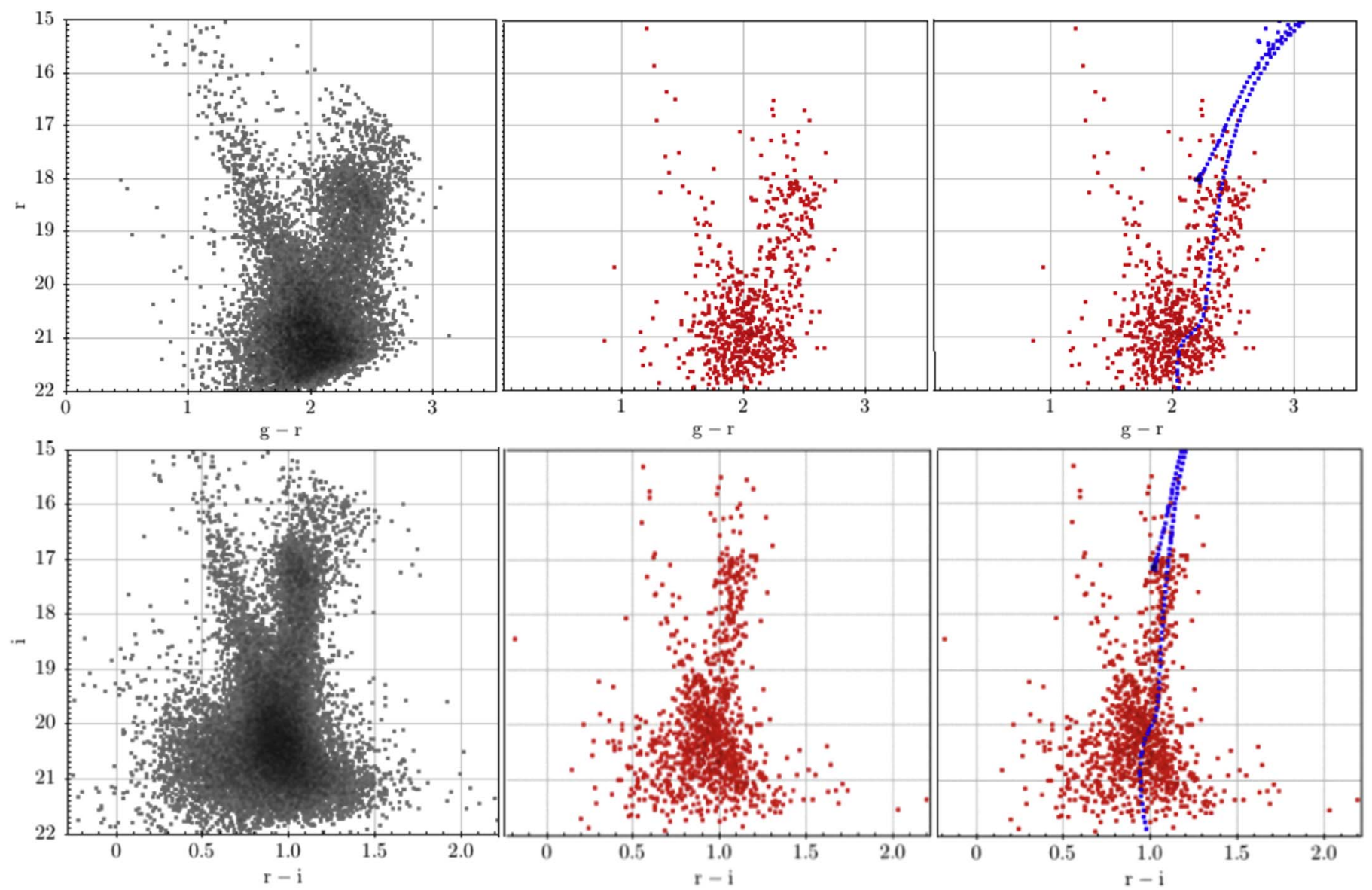

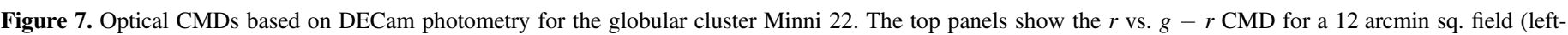

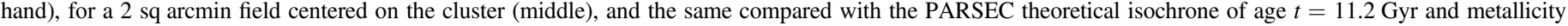

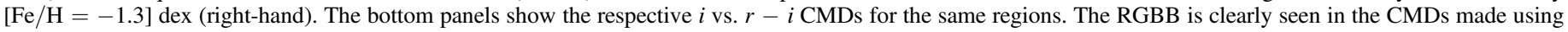
the DECam photometry.

coadding the fluxes of the stars yields total apparent magnitudes $J_{\text {total }}=7.6$ and $K s_{\text {total }}=6.4 \mathrm{mag}$. By correcting for absorption $\left(A_{K s}=0.47\right)$, we obtain $K s_{0}=5.9 \mathrm{mag}$. The intrinsic mean optical near-IR color of GCs in the Milky Way and M31 is known to be $(V-K)_{0}=2.2 \pm 0.1 \mathrm{mag}$ (e.g., Grasdalen 1974; Cohen 1993). By adopting this value (and ignoring the small difference between the $K$ and $K s$-bands), we obtain a total optical magnitude $V_{0}=8.1 \mathrm{mag}$ and absolute magnitude of $M_{V}=-6.2 \mathrm{mag}$ for a distance of $D=7.4 \mathrm{kpc}$. We are aware that this is a very uncertain estimate because of the high background and differential reddening, and so we conservatively adopt an error of $\sigma \sim 0.5 \mathrm{mag}$ because the absolute magnitude depends not only on the distance but also on the extinction and on the statistical decontamination of the CMD. In conclusion, we find that Minni 22 is a low-luminosity Galactic GC.

\section{Optical Photometry with DECam and Gaia}

We matched the VVV near-IR stellar sources to the optical DECaPS sources from Schlafly et al. (2017). Because these are crowded fields, we matched the stellar positions to within 0.6 arcsec (larger match tolerances like 1 arcsec lead to too many duplicate sources). The combined VVV-DECaPS multicolor photometry is available in machine readable format in Table 2. Figure 7 shows the optical $r$ versus $g-r$ and $i$ versus $r-i$ CMDs based on DECam photometry for Minni 22, as well as the
CMDs for a 12 arcmin sq. field compared with a small 2 sq. arcmin field centered on the cluster. This figure also presents the $r$ versus $g-r$ and $i$ versus $r-i$ CMDs for the cluster region in comparison with PARSEC theoretical isochrones. The RGBB is clearly seen in all the CMDs constructed using the DECam photometry for the different combinations of the grizY filters (no $u$-band photometry is available).

From the optical CMDs, the measured RGBB using DECaPS photometry (Schlafly et al. 2017) yields the following mean $\mathrm{AB}$ magnitudes: $r_{\mathrm{RGBB}}=18.2, g_{\mathrm{RGBB}}=20.6, i_{\mathrm{RGBB}}=$ $17.1, z_{\mathrm{RGBB}}=16.3$, and $Y_{\mathrm{RGBB}}=16.0 \mathrm{mag}$, and mean colors: $g-r_{\mathrm{RGBB}}=2.4, g-i_{\mathrm{RGBB}}=3.5, g-Y_{\mathrm{RGBB}}=4.6, r-$ $i_{\mathrm{RGBB}}=1.08, i-z_{\mathrm{RGBB}}=0.75$, and $z-Y_{\mathrm{RGBB}}=0.34 \mathrm{mag}$. For $K s_{\mathrm{RGBB}}=13.30 \mathrm{mag}$ from the VVV photometry, the optical-infrared colors of the RGBB are: $g-K s_{\mathrm{RGBB}}=$ $7.5, i-K s_{\mathrm{RGBB}}=4.0 \mathrm{mag}$.

We have also fitted the Bressan et al. (2012) isochrones, adding the fits to the combined optical near-IR CMDs of Figures 7 and 8. Figure 8 shows optical-IR CMDs compared with theoretical isochrones. The DECaPS photometry of Schlafly et al. (2017) in itself is deeper than the main-sequence TO. Unfortunately, the combination of optical near-IR CMD barely reaches the main sequence TO. In this case, the fits can only be used to rule out younger ages but they cannot be used to accurately measure the cluster's age. The isochrone in the CMD $(g-K s, K s)$ has an insignificant offset from the main locus, which depends on the distance, extinction and color, as 

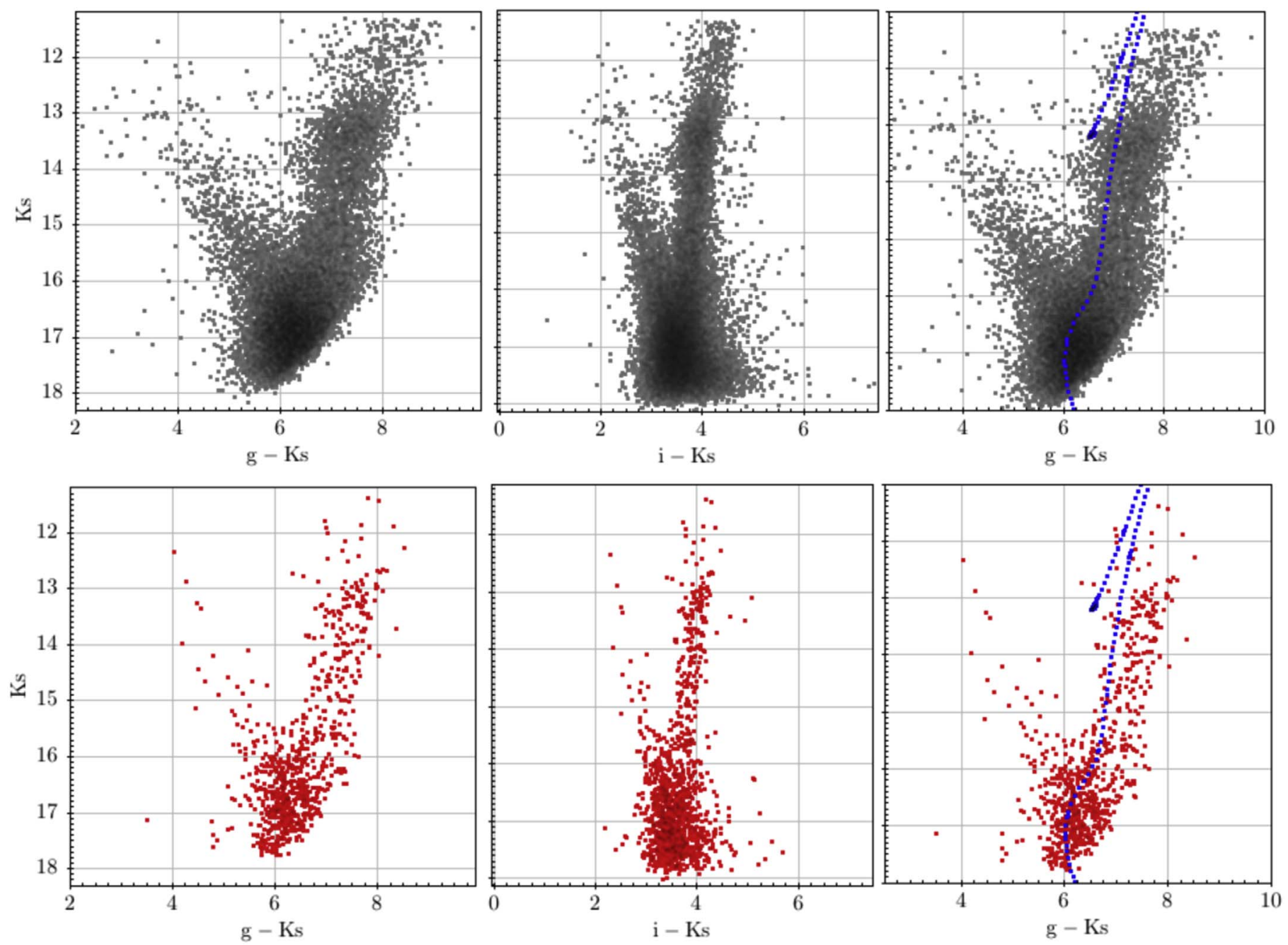

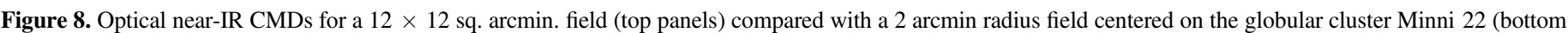

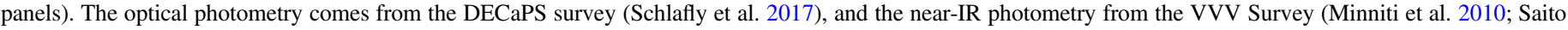

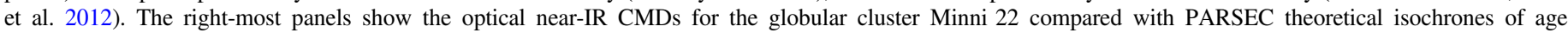
$t=11.2 \mathrm{Gyr}$ and and metallicity $[\mathrm{Fe} / \mathrm{H}=-1.3]$ dex (right-hand).

well as on the particular reddening law adopted. All of our CMDs are consistent with an age of $t>10 \mathrm{Gyr}$, as suggested by the presence of RR Lyrae stars.

The large wavelength baseline helps to discriminate the background field stars. Note that the DECam photometry is in $\mathrm{AB}$ magnitudes, while the VVV photometry is in Vega magnitudes. The PARSEC isochrones that are used here correspond to these magnitude scales.

Given the quality of the combined optical and near-IR data, we conclude that at present the best way to confirm these objects as GCs are CMDs based on DECaPS + VVV photometry.

The Gaia space mission also obtained optical photometry of the region in the broad $G$-band (Perryman et al. 2001; Jordi et al. 2010; Gaia Collaboration et al. 2016, 2018). ${ }^{17}$ Again, because these are crowded fields, we matched the stellar positions to within 0.6 arcsec. In Figure 9, we compare the near-IR $K s$ versus $G-K s$ CMD for Minni 22 with the corresponding optical near-IR CMD. Both CMDs look similar,

\footnotetext{
${ }^{17}$ We consider Gaia DR1 data. The future DR2 data will be more complete, particularly in the bulge regions where detections are sometimes thrown because of buffer filling.
}

although the effect of differential reddening can be more clearly observed in the optical photometry. This results in a wider disk main sequence and cluster RGB. As shown in Figure 9, the Gaia photometry is not as deep as the DECam photometry, which reaches the cluster TO. However, this figure illustrates that Gaia would be able to obtain PMs for the cluster red giants.

\section{VVV PMs}

The PM vector diagram can be used to decontaminate the CMDs of the GCs in the Galactic bulge (see Libralato et al. 2015; Contreras Ramos et al. 2017). Recently, Smith et al. (2017) made a full astrometric catalog of the VVV survey (VIRAC), measuring PMs for stellar objects in the VVV nearIR images down to $K s \sim 17 \mathrm{mag}$, including the region of Minni 22. Their PMs have a median uncertainty of $\sigma=0.67 \mathrm{mas} \mathrm{yr}^{-1}$ in the $11<K s<14 \mathrm{mag}$ range, which includes the RGB of Minni 22. These accurate PMs are very useful to separate bulge stars from foreground stars in the Galactic disk (Smith et al. 2018).

The PM vector diagram shown in Figure 10 for the $2^{\prime} \times 2^{\prime}$ region of this GC shows that the mean cluster motion is not 


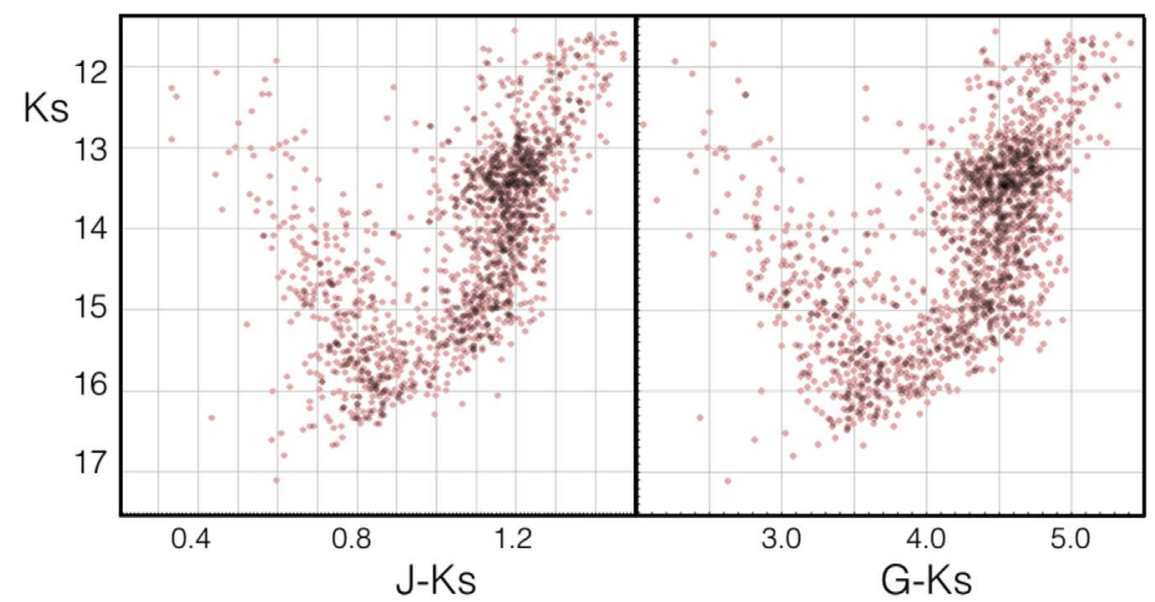

Figure 9. Near-IR CMD (left-hand) compared with the optical near-IR CMD (right-hand) for the globular cluster Minni 22. The optical photometry comes from Gaia and the near-IR photometry from the VVV Survey. Unfortunately, the Gaia photometry is not as deep as the DECam photometry that reaches the cluster TO. However, this figure illustrates that Gaia would be able to obtain PMs for the cluster red giants.
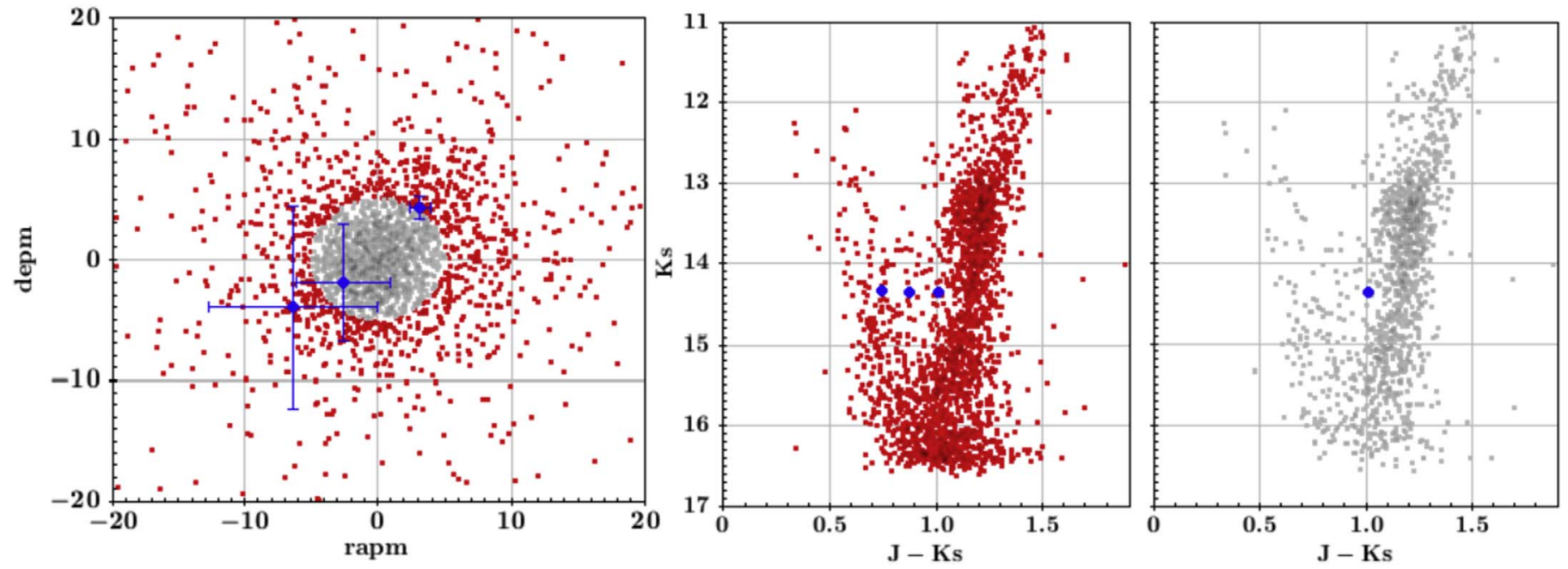

Figure 10. Left-hand panel: Ground based PMs measured using VVV Survey images (see Smith et al. 2017). The Minni 22 cluster RR Lyrae from Table 1 are plotted in blue. Middle panel: Near-IR CMD for a 2 arcmin sq. field centered in the GC Minni 22 for all the stars with measured PMs. Right-hand panel: Near-IR CMD for the same region but using only PM selected stars (stars inside the gray circle in the left-hand panel). The PM selection efficiently cleans the RGB and eliminates most foreground disk stars but loses stars near the main-sequence TO region.

very different from that of the bulk of the bulge field stars. Nevertheless, the PMs still provide a useful way of cleaning the CMDs in the Galactic bulge direction because the foreground disk stars exhibit an asymmetric drift. The PM cleaned CMD, which is shown in the right-hand panel of Figure 10, exhibits a tighter RGB and a prominent RGBB. The location of these GC main features match the location of the features seen in the CMDs obtained using the statistical background decontamination (Figure 6). Unfortunately, the PM selection does not reach the same depth as the VVV CMDs in these fields, which go as far as $1.5 \mathrm{mag}$ deeper, down to the MS TO. The PM errors increase as the magnitudes become fainter, as shown in Figure 5 of Smith et al. (2017). Only the most accurate measurements were used (with reliable flag $=1$ ), thus eliminating the faintest sources. In addition, the cluster CMD in Figure 10 appears to best show a blue HB component that is similar to that of the comparable template GC NGC 6642 (Figure 4), which further corroborates the metal-poor GC nature of Minni 22.

\section{Conclusions}

Based on different lines of evidence, such as combined optical and near-IR photometry, the presence of RR Lyrae and the PM of cluster stars, we conclude that Minni 22 is a genuine bulge GC. Although this might be considered overkill, it is necessary to confirm the physical reality of a new GC candidate due to a variety of troubles that arise when seeking GCs in the bulge direction, such as high interstellar extinction, foreground and background stellar contamination and crowding.

A summary of the cluster parameters is given in Table 3 . The near-IR CMD of the GC Minni 22 is similar to that of the metal-poor Galactic GC NGC 6642. The optical CMDs also look like metal-poor GCs. The best fit with a theoretical stellar isochrone yields an age of $t=11.2 \mathrm{Gyr}$, and a metallicity $[\mathrm{Fe} / \mathrm{H}]=1.3$ dex, Minni 22 hosts three RR Lyrae. This fact not only indicates its GC nature but also that it corresponds to metal-poor stellar populations. We point out that although the co-existence of multiple RR Lyrae within a small volume is a valid confirmation of GC status, it is not a pre-requisite (not all 
Table 3

Measured Parameters for the Globular Cluster Minni 22

\begin{tabular}{lccc}
\hline \hline Parameter & Value & Error & Comment \\
\hline R.A.(J2000) & $17: 48: 49$ & $30 \mathrm{arcsec}$ & Center from the density map of red giants \\
Decl.(J2000) & $-33: 03: 03$ & $30 \mathrm{arcsec}$ & Center from the density map of red giants \\
$r_{h}$ & $1.1 \mathrm{arcmin}$ & $0.3 \mathrm{arcmin}$ & Half light radius from star counts \\
$A_{K s}$ & $0.47 \mathrm{mag}$ & $0.05 \mathrm{mag}$ & Estimated from Schlafly \& Finkbeiner (2011) \\
$(m-M)_{0}$ & $14.30 \mathrm{mag}$ & $0.08 \mathrm{mag}$ & PLZ relations from Muraveva and Navarrete \\
Distance & $7.4 \mathrm{kpc}$ & $0.3 \mathrm{kpc}$ & Mean distance of RRab \\
Age & $\sim 11.2 \mathrm{Gyr}$ & $1.0 \mathrm{Gyr}$ & From the PARSEC isochrones \\
{$[\mathrm{Fe} / \mathrm{H}]$} & $-1.3 \mathrm{dex}$ & $0.3 \mathrm{dex}$ & Isochrones and presence of RRab \\
$M_{V}$ & $-6.2 \mathrm{mag}$ & $0.5 \mathrm{mag}$ & Coadded stars in the decontaminated CMD \\
\hline
\end{tabular}

GCs host RR Lyrae). The presence of RR Lyrae also indicates that this is an old cluster (with age $>10 \mathrm{Gyr}$ ). We determine for the GC Minni 22 an accurate distance $D=7.4 \pm 0.3 \mathrm{kpc}$ from the RRab stars. PMs have also been used to clean the cluster RGB to verify the position of its RGBB and to confirm the presence of an extended HB. We also estimate that this is a low-luminosity Galactic GC, with $M_{V}=-6.2 \mathrm{mag}$.

This is the first IR GC candidate that has been confirmed with optical DECaPS grizY and Gaia $G$-band photometry. Based on this study, we conclude that one of the most powerful ways to confirm new GC candidates in the Galactic bulge is to use a combination of optical and near-IR photometries.

The successful confirmation of Minni 22 as a genuine GC indicates that many of the new candidates that are being discovered in the Galactic bulge (Minniti et al. 2017) are probably also real GCs. Is Minni 22 representative of a new population of low-luminosity old GCs in the bulge? Are we seeing the core of a dissolving/disrupted GC? These questions remain open and Minni 22 might well be a good candidate for a low-luminosity dissolving cluster in the innermost part of our Galaxy.

We gratefully acknowledge data from the ESO Public Survey program ID 179.B-2002 taken with the VISTA telescope, and products from the Cambridge Astronomical Survey Unit (CASU). DECaPS is based on observations at Cerro Tololo Inter-American Observatory, National Optical Astronomy Observatory (NOAO Prop. ID: 2014A-0429, 2016A-0327, and 2016B-0279; PI: Finkbeiner), which is operated by the Association of Universities for Research in Astronomy (AURA) under a cooperative agreement with the National Science Foundation. Support for this work was provided by the BASAL Center for Astrophysics and Associated Technologies (CATA) through grant PFB-06, and by the Ministry for the Economy, Development and Tourism, Programa Iniciativa Cientifica Milenio grant IC120009, awarded to the Millennium Institute of Astrophysics (MAS). E.S. acknowledges support for this work provided by NASA through Hubble Fellowship grant HST-HF2-51367.001-A awarded by the Space Telescope Science Institute, which is operated by the Association of Universities for Research in Astronomy, Inc., for NASA, under contract NAS 5-26555. D.M. acknowledges support from FONDECYT Regular grant No. 1170121. J.A-G. acknowledges support by FONDECYT Iniciacion 11150916, and by the Ministry of Education through grant ANT-1656. M.H. also acknowledges support from
BASAL CATA through grant PFB-06. R.K.S. acknowledges support from CNPq/Brazil through projects 308968/2016-6 and $421687 / 2016-9$, and C. B. and E. B. also thank the support from $\mathrm{CNPq} /$ Brazil.

\section{ORCID iDs}

Dante Minniti (iD https://orcid.org/0000-0002-7064-099X

E. F. Schlafly (i) https://orcid.org/0000-0002-3569-7421

Javier Alonso-García (i) https://orcid.org/0000-0003-

3496-3772

Vittorio F. Braga (1D https://orcid.org/0000-0001-7511-2830

Valentin D. Ivanov (i) https://orcid.org/0000-0002-5963-1283

Phillip W. Lucas (D) https://orcid.org/0000-0002-8872-4462

Roberto K. Saito (10) https://orcid.org/0000-0001-6878-8648

\section{References}

Alonso-García, J., Dékány, I., Catelan, M., et al. 2015, AJ, 149, 99 Alonso-García, J., Minniti, D., Catelan, M., et al. 2017, ApJL, 849, L13 Barbuy, B., Bica, E., Ortolani, S., \& Bonatto, C. 2006, A\&A, 449, 1019 Barbuy, B., Zoccali, M., \& Ortolani, S. 2009, A\&A, 507, 405 Bonatto, C., Bica, E., Ortolani, S., \& Barbuy, B. 2007, MNRAS, 381, 45 Bressan, A., Marigo, P., Girardi, L., et al. 2012, MNRAS, 427, 127 Cardelli, J. A., Clayton, G. C., \& Mathis, J. S. 1989, ApJ, 345, 245 Catelan, M., \& Smith, H. A. 2015, Pulsating Stars (New York: Wiley-VCH) Chen, C. W., \& Chen, W. P. 2010, ApJ, 721, 1790

Cohen, J. G. 1993, in ASP Conf. Ser. 48, The Globular Clusters-galaxy Connection, ed. G. H. Smith \& J. P. Brodie (San Francisco, CA: ASP), 438

Contreras Ramos, R., Zoccali, M., Rojas, F., et al. 2017, A\&A, 608, A140

Emerson, J., \& Sutherland, W. 2010, Msngr, 139, 2

Fitzpatrick, E. L. 1999, PASP, 111, 63

Gaia Collaboration, Babusiaux, C., van Leeuwen, F., et al. 2018, A\&A, 616, A10

Gaia Collaboration, Brown, A. G. A., Vallenari, A., et al. 2016, A\&A, 595, A2

Gonzalez, O. A., Rejkuba, M., Zoccali, M., et al. 2012, A\&A, 543, 13

Grasdalen, G. L. 1974, AJ, 79, 1047

Harris, W. E. 2010, arXiv:1012.3224

Irwin, M. J., Lewis, J., Hodgkin, S., et al. 2004, Proc. SPIE, 5493, 411

Jordi, C., Gebran, M., Carrasco, J. M., et al. 2010, A\&A, 523, A48

Kim, J.-W., Kang, A, Rhee, J., et al. 2006, A\&A, 459, 499

Koposov, S., de Jong, J. T. A., Belokurov, V., et al. 2007, ApJ, 669, 337

Libralato, M., Bellini, A., Bedin, L. R., et al. 2015, MNRAS, 450, 1664

Marigo, P., Girardi, L., Bressan, A., et al. 2017, ApJ, 835, 77

Minniti, D. 1995a, A\&A, 303, 468

Minniti, D., Geisler, D., Alonso-García, J., et al. 2017, ApJL, 849, L24

Minniti, D., Lucas, P. W., Emerson, J. P., et al. 2010, NewA, 15, 433

Muraveva, T., Palmer, M., Clementini, G., et al. 2015, ApJ, 807, 127

Navarrete, C., Catelan, M., Contreras Ramos, R., et al. 2017, A\&A, 604, 120 
Oosterhoff, P. T. 1939, Obs, 62, 104

Palma, T., Minniti, D., Dekany, I., Clariá, J. J., \& Alonso-García, J. 2016, NewA, 49, 50

Perryman, M. A. C., de Boer, K. S., Gilmore, G., et al. 2001, A\&A, 369, 339

Pietrukowicz, P., Kozowski, S., Skowron, J., et al. 2015, ApJ, 811, 113

Saito, R. K., Hempel, M., Minniti, D., et al. 2012, A\&A, 537, A107

Schlafly, E. F., \& Finkbeiner, D. P. 2011, ApJ, 737, 103
Schlafly, E. F., Peek, J. E. G., Finkbeiner, D. P., \& Green, G. M. 2017, ApJ, 838,36

Smith, L. C., Lucas, P. W., Kurtev, R., et al. 2018, MNRAS, 474, 1826

Suntzeff, N. B., Kinman, T. D., \& Kraft, R. P. 1991, ApJ, 367, 528

Valenti, E., Ferraro, F. R., \& Origlia, L. 2004, MNRAS, 351, 1204

Valenti, E., Ferraro, F. R., \& Origlia, L. 2010, MNRAS, 402, 1729

White, R. E., \& Shawl, S. J. 1987, ApJ, 317, 246 